\title{
Hamilton-Jacobi flows with nowhere differentiable initial data
}

\author{
Yasuhiro Fujita ${ }^{1}\left[\right.$ - Antonio Siconolfi ${ }^{2} \cdot$ Norikazu Yamaguchi $^{1}$ \\ Received: 24 August 2021 / Revised: 13 December 2021 / Accepted: 20 December 2021 / \\ Published online: 15 February 2022 \\ (c) The Author(s) 2022
}

\begin{abstract}
We deal with a sort of inverse problem. Namely, we aim at deriving features of initial data of an Hamilton-Jacobi flow starting from specific assumptions on the flow. To this scope, we introduce a class of nowhere differentiable functions, and characterize a function in this class through a property of the Hamilton-Jacobi flow issued from this function. This analysis shows that, despite the regularizing effect of the flow, nowhere differentiability of initial data can be nevertheless detected looking at the flow for positive times.
\end{abstract}

Mathematics Subject Classification 26A27 · 26A99 $\cdot$ 39B22

\section{Introduction}

We call partition of $[0,1]$ any finite strictly increasing sequence $a_{k}, k=0,1, \cdots, N$ of points in $[0,1]$ with $a_{0}=0, a_{N}=1$. Given a partition $\Gamma=\left\{a_{k}\right\}_{k=0}^{N}$, we set

$$
|\Gamma|=\max _{1 \leq k \leq N}\left(a_{k}-a_{k-1}\right) .
$$

Communicated by Giga.

Fujita is supported in part by JSPS KAKENHI Nos. 18K03360 and $21 \mathrm{~K} 03322$.

Yasuhiro Fujita

yfujita@sci.u-toyama.ac.jp

Antonio Siconolfi

siconolf@mat.uniroma1.it

Norikazu Yamaguchi

norikazu@edu.u-toyama.ac.jp

1 University of Toyama, 3190 Gofuku, Toyama-shi, Toyama 930-8555, Japan

2 Sapienza Università di Roma, Piazzale Aldo Moro 5, 00185 Roma, Italy 
Set $\mathbb{N}_{0}=\{0,1,2, \cdots\}$.

Definition 1.1 A sequence $\left\{\Gamma_{n}\right\}_{n \in \mathbb{N}_{0}}$ of partitions of $[0,1]$ is called admissible if the following hold:

(D1) $\Gamma_{0}=\{0,1\}$.

(D2) For each $n \in \mathbb{N}_{0}, \Gamma_{n} \subset \Gamma_{n+1}$.

(D3) $\left|\Gamma_{n}\right| \rightarrow 0$ as $n \rightarrow \infty$.

A typical example of admissible sequence of partitions of $[0,1]$ is given by

$$
\Gamma_{n}=\left\{\frac{k}{r^{n}} \mid k \in \mathbb{N}_{0}, 0 \leq k \leq r^{n}\right\}, \quad n \in \mathbb{N}_{0}
$$

where $r$ is an integer such that $r \geq 2$.

We denote by $C_{p}(\mathbb{R})$ the set of all continuous and 1-periodic functions $f$ on $\mathbb{R}$ satisfying $f(0)=0$. For a given function $f \in C_{p}(\mathbb{R})$, we define

$$
H_{t} f(x):=\inf _{y \in \mathbb{R}} q_{f}(t, x ; y), \quad(t, x) \in(0, \infty) \times \mathbb{R},
$$

where $\left\{q_{f}(t, x ; y)\right\}_{y \in \mathbb{R}}$ is the family of parabolas defined by

$$
q_{f}(t, x ; y)=f(y)+\frac{1}{2 t}(x-y)^{2}, \quad(t, x, y) \in(0, \infty) \times \mathbb{R} \times \mathbb{R} .
$$

The function $H_{t} f$ is called the Moreau envelope of $f$, which is a special case of inf-convolution. It is known that $H_{t} f$ is a unique viscosity solution to the following Cauchy problem of the Hamilton-Jacobi equation in the class of bounded and uniformly continuous functions on $[0, T) \times \mathbb{R}$ for each $T \in(0, \infty)$ :

$$
\begin{array}{ll}
\frac{\partial u}{\partial t}(t, x)+\frac{1}{2}\left(\frac{\partial u}{\partial x}(t, x)\right)^{2}=0, & (t, x) \in(0, T) \times \mathbb{R}, \\
u(0, x)=f(x), & x \in \mathbb{R}
\end{array}
$$

(cf. [11, Propositions A.2 and A.4]).

We introduce the following condition for the Hamilton-Jacobi flow $\left\{H_{t} f\right\}$ :

(HJ) There exist a constant $\theta \in(0, \infty)$ and an admissible sequence $\left\{\Gamma_{n}\right\}$ of partitions of the interval $[0,1]$ satisfying

$$
H_{t} f(x)=\min _{y \in \Gamma_{n}} q_{f}(t, x ; y), \quad(t, x) \in\left[\frac{\left|\Gamma_{n}\right|}{2 \theta}, \infty\right) \times[0,1]
$$

for every $n \in \mathbb{N}_{0}$.

We immediately derive from (1.3) that any $H_{t} f$ satisfying (HJ) is piecewise quadratic on the interval $[0,1]$ for all $t>0$, since $\left|\Gamma_{n}\right| \rightarrow 0$ as $n \rightarrow \infty$. Our aim is to determine all initial data $f \in C_{p}(\mathbb{R})$ such that $\left\{H_{t} f\right\}$ satisfies $(\mathrm{HJ})$. For this purpose, we introduce the following class: 
Definition 1.2 (The class $\mathcal{R}$ ) Let $\theta \in(0, \infty)$. A function $f \in C_{p}(\mathbb{R})$ belongs to $\mathcal{R}_{\theta}$ if there exists an admissible sequence $\left\{\Gamma_{n}\right\}$ of partitions $\Gamma_{n}=\left\{a_{k}^{(n)}\right\}_{k=0}^{N_{n}}$ of $[0,1]$ satisfying

$$
\frac{f\left(a_{k}^{(n)}\right)-f(x)}{a_{k}^{(n)}-x}-\frac{f(x)-f\left(a_{k-1}^{(n)}\right)}{x-a_{k-1}^{(n)}} \leq-\theta, \quad x \in\left(a_{k-1}^{(n)}, a_{k}^{(n)}\right), k \in\left\{1,2, \cdots, N_{n}\right\}
$$

for each $n \in \mathbb{N}_{0}$. We set $\mathcal{R}=\bigcup_{\theta>0} \mathcal{R}_{\theta}$.

The above class $\mathcal{R}$ reduces to $\mathcal{P}=\bigcup_{\theta>0} \mathcal{P}_{\theta}$, already defined in [7], if $\left\{\Gamma_{n}\right\}$ is taken as in (1.1). We recall that each function of $\mathcal{P}_{\theta}$ is nowhere differentiable, and that it has been proven in [7] that if $f \in \mathcal{P}_{\theta}$, for some $\theta \in(0, \infty)$, then $\left\{H_{t} f\right\}$ satisfies (HJ) with this $\theta$ and $\left\{\Gamma_{n}\right\}$ taken as in (1.1). Conversely, if $\left\{H_{t} f\right\}$ satisfies (1.3), for $f \in C_{p}(\mathbb{R})$, with some $\theta>0$ and $\left\{\Gamma_{n}\right\}$ of the form (1.1), we have already in [7] a partial result showing that $f$ is non-differentiable at each point of a dense subset of $[0,1]$. We make here a step forward, as illustrated by the forthcoming Proposition 1.4 and Theorem 1.5 .

Functions in $\mathcal{R}$, for general admissible sequences of partitions of the interval $[0,1]$, satisfy the following relevant properties:

Proposition 1.3 Each function in $\mathcal{R}$ is nowhere differentiable on $\mathbb{R}$.

Proposition 1.4 If $f \in \mathcal{R}$ then $\left\{H_{t} f\right\}$ satisfies $(\mathrm{HJ})$.

Proofs of the above results will be given in Sect. 3 .

For a given admissible sequence $\left\{\Gamma_{n}\right\}$ of partitions of the interval $[0,1]$, we consider the following condition:

(A1) There exists a constant $\delta \in(0,1]$ such that

$$
\left|\Gamma_{n}\right| \leq \frac{1}{\delta} \min \left\{|x-y| \mid x, y \in \Gamma_{n}, x \neq y\right\}, \quad n \in \mathbb{N}_{0} .
$$

Note that when $\Gamma_{n}$ is given by (1.1), (A1) is fulfilled with $\delta=1$. As for some result for (A1) and other examples of admissible sequences of partitions of [0,1] satisfying (A1) or not satisfying (A1), see Sect. 4.

Our main result is:

Theorem 1.5 Let $f \in C_{p}(\mathbb{R})$. Assume that $\left\{H_{t} f\right\}$ satisfies $(\mathrm{HJ})$ and that the admissible sequence $\left\{\Gamma_{n}\right\}$ of partitions of $[0,1]$ appearing in $(\mathrm{HJ})$ satisfies $(\mathrm{A} 1)$. Then $f \in \mathcal{R}$. In particular, $f$ is nowhere differentiable on $\mathbb{R}$.

Condition (A1) seems unavoidable for proving Theorem 1.5. Proposition 1.4 and Theorem 1.5 together say that the Hamilton-Jacobi property (HJ) almost characterizes functions in $\mathcal{R}$, although we need adding (A1) in Theorem 1.5. In Theorem 1.5, we perform a sort of inverse analysis for the Hamilton-Jacobi flow, in the sense that we determine some features of initial data starting from properties of the corresponding 
flows. As for another type of inverse problems for Hamilton-Jacobi equations, see $[4,13]$ etc.

In Sect. 3, we give a more general version of condition (1.3) in (HJ), showing that Theorem 1.5 still holds even if (1.3) in (HJ) is replaced by

$$
H_{t_{n}} f(x)=\min _{y \in \Gamma_{n}} q_{f}\left(t_{n}, x ; y\right), \quad x \in[0,1]
$$

for some positive sequence $\left\{t_{n}\right\}$ converging to 0 as $n \rightarrow \infty$ (see Theorem 3.16).

Next, we provide examples of functions belonging to the class $\mathcal{R}$ for a better understanding of the theory. In [7], we already gave several examples of functions in the class $\mathcal{P}$, such as the generalized Takagi function $\tau_{r}$, with an integer $r$ such that $r \geq 2$. It is defined by

$$
\tau_{r}(x)=\sum_{n=0}^{\infty} d\left(x, \Gamma_{n}\right), \quad x \in[0,1]
$$

with $\Gamma_{n}$ of the form (1.1). Here $d(\cdot, E)$ is the Euclidean distance function from a subset $E$ of $\mathbb{R}$, that is,

$$
d(x, E)=\min \{|x-z| \mid z \in E\}, \quad x \in \mathbb{R} .
$$

Note that $\tau_{r}$ can be equivalently written as

$$
\tau_{r}(x)=\sum_{n=0}^{\infty} \frac{1}{r^{n}} d\left(r^{n} x, \mathbb{Z}\right), \quad x \in[0,1] .
$$

When $r=2$, it is just the classical Takagi function (see [1,12,14]).

In this paper, adapting the procedure given in [7], we systematically construct, for a given admissible sequence of partitions $\left\{\Gamma_{n}\right\}$ of $[0,1]$, examples of functions in the class $\mathcal{R}$. As a special case, this class includes the functions

$$
f(x)=\sum_{n=0}^{\infty} d\left(x, \Gamma_{n}\right), \quad x \in[0,1],
$$

where $\left\{\Gamma_{n}\right\}$ is any admissible sequence of partitions of $[0,1]$ with $\sum_{n=0}^{\infty}\left|\Gamma_{n}\right|<+\infty$. The function of (1.5) was studied by Ferrera and Gómez Gil [6] (see Remark 3.4).

The paper is organized as follow: In Sect. 2, we give some preliminaries. In Sect. 3, we state and prove our main results. Theorem 1.5 is proved here. In Sect. 4 , we give examples of admissible sequences of partitions of $[0,1]$ satisfying (A1) and functions belonging to $\mathcal{R}$.

\section{Preliminaries}

We first recall some definitions we need in what follows. Given a lower semicontinuous function $f$ defined on $\mathbb{R}$, we say that a $C^{1}$ function $\psi$ is subtangent to $f$ at a point 
$x_{0}$ if $\psi\left(x_{0}\right)=f\left(x_{0}\right)$ and $\psi(x) \leq f(x)$ in a neighborhood of $x_{0}$. The subdifferential $D^{-} f\left(x_{0}\right)$ of $f$ at $x_{0}$ is defined as follows:

$$
\begin{aligned}
D^{-} f\left(x_{0}\right) & =\left\{p \in \mathbb{R} \mid \liminf _{y \rightarrow x_{0}} \frac{f(y)-f\left(x_{0}\right)-p\left(y-x_{0}\right)}{\left|y-x_{0}\right|} \geq 0\right\} \\
& =\left\{p \in \mathbb{R} \mid p=\psi^{\prime}\left(x_{0}\right) \text { with } C^{1} \text { subtangent } \psi \text { to } f \text { at } x_{0}\right\}
\end{aligned}
$$

This set is closed, convex, possibly empty. It is clear that if $D^{-} f\left(x_{0}\right)$ is empty or multivalued, then $f$ is not differentiable at $x_{0}$ (see [2, p.29] and [3, p.50]).

It is now convenient to introduce the Dini derivatives of a function $f: \mathbb{R} \rightarrow \mathbb{R}$ at a point $x_{0}$, and to put them in relation with the subdifferential $D^{-} f\left(x_{0}\right)$. Dini derivatives at $x_{0}$ are four, possibly infinite:

$$
\begin{aligned}
& \mathfrak{D}_{-} f\left(x_{0}\right)=\liminf _{h \rightarrow 0^{-}} \frac{f\left(x_{0}+h\right)-f\left(x_{0}\right)}{h}, \\
& \mathfrak{D}^{-} f\left(x_{0}\right)=\limsup _{h \rightarrow 0^{-}} \frac{f\left(x_{0}+h\right)-f\left(x_{0}\right)}{h}, \\
& \mathfrak{D}_{+} f\left(x_{0}\right)=\liminf _{h \rightarrow 0^{+}} \frac{f\left(x_{0}+h\right)-f\left(x_{0}\right)}{h}, \\
& \mathfrak{D}^{+} f\left(x_{0}\right)=\limsup _{h \rightarrow 0^{+}} \frac{f\left(x_{0}+h\right)-f\left(x_{0}\right)}{h} .
\end{aligned}
$$

It is clear that $f$ is differentiable at $x_{0}$ if and only if all the Dini derivatives are finite and coincide. For $a \in \mathbb{R} \cup\{ \pm \infty\}$ and $b \in \mathbb{R} \cup\{ \pm \infty\}$, we define the set $\llbracket a, b \rrbracket$ by

$$
\llbracket a, b \rrbracket= \begin{cases}{[a, b]} & \text { if } a, b \in \mathbb{R} \text { and } a \leq b, \\ {[a, \infty)} & \text { if } a \in \mathbb{R} \text { and } b=+\infty \\ (-\infty, b] & \text { if } a=-\infty, \text { and } b \in \mathbb{R}, \\ \mathbb{R} & \text { if } a=-\infty, b=+\infty, \\ \emptyset & \text { otherwise. }\end{cases}
$$

Proposition 2.1 Given a lower semicontinuous function $f: \mathbb{R} \rightarrow \mathbb{R}$ and $x_{0} \in \mathbb{R}$, we have

$$
D^{-} f\left(x_{0}\right)=\llbracket \mathfrak{D}^{-} f\left(x_{0}\right), \mathfrak{D}_{+} f\left(x_{0}\right) \rrbracket .
$$

Proof Given $p \in \mathbb{R}$, we have

$$
\begin{gathered}
\liminf _{y \rightarrow x_{0}-} \frac{f(y)-f\left(x_{0}\right)-p\left(y-x_{0}\right)}{\left|y-x_{0}\right|}=\liminf _{y \rightarrow x_{0}-}\left(-\frac{f(y)-f\left(x_{0}\right)}{y-x_{0}}+p\right) \\
=-\limsup _{y \rightarrow x_{0}-} \frac{f(y)-f\left(x_{0}\right)}{y-x_{0}}+p=-\mathfrak{D}^{-} f\left(x_{0}\right)+p
\end{gathered}
$$

and

$\liminf _{y \rightarrow x_{0}+} \frac{f(y)-f\left(x_{0}\right)-p\left(y-x_{0}\right)}{\left|y-x_{0}\right|}=\liminf _{y \rightarrow x_{0}+}\left(\frac{f(y)-f\left(x_{0}\right)}{y-x_{0}}-p\right)=\mathfrak{D}_{+} f\left(x_{0}\right)-p$. 
From these equalities, it is not difficult to show (2.2). The proof is complete.

The following result is obvious.

Lemma 2.2 Let $a, b, \theta \in \mathbb{R}$ with $a<b$ and $\theta>0$. Then, for a function $f:[a, b] \rightarrow$ $\mathbb{R}$, the inequality

$$
\frac{f(b)-f(x)}{b-x}-\frac{f(x)-f(a)}{x-a} \leq-\theta, \quad x \in(a, b)
$$

is equivalent to the inequality

$$
f(x) \geq \frac{\theta}{b-a}(x-a)(b-x)+\frac{1}{b-a}[(b-x) f(a)+(x-a) f(b)], x \in(a, b) .
$$

The next lemma can be proved similarly to [7, Proposition 2.2]. For given $a, b, t \in \mathbb{R}$ with $a<b$ and $t>0$, we denote by $x_{a, b}(t)$ the unique point of intersection between $q_{f}(t, \cdot ; a)$ and $q_{f}(t, \cdot ; b)$, that is,

$$
x_{a, b}(t)=\frac{a+b}{2}+t \frac{f(b)-f(a)}{b-a} .
$$

Lemma 2.3 Let $a, b, t \in \mathbb{R}$ with $a<b$ and $t>0$. Then the inequality

$$
\min \left\{q_{f}(t, x ; a), q_{f}(t, x ; b)\right\} \leq q_{f}(t, x ; y), \quad x \in \mathbb{R}, y \in(a, b)
$$

is equivalent to the inequality

$$
\frac{f(b)-f(y)}{b-y}-\frac{f(y)-f(a)}{y-a} \leq-\frac{b-a}{2 t}, \quad y \in(a, b) .
$$

Proof A geometrical investigation shows that inequality (2.4) holds if and only if the inequality $x_{y, b}(t) \leq x_{a, y}(t)$ holds for all $y \in(a, b)$. It is easy to see that this inequality is equivalent to that in $(2.5)$.

We record for later use:

Lemma 2.4 Let $f \in C_{p}(\mathbb{R})$ and $a, b, p, t \in \mathbb{R}$ with $t>0$. Then $q_{f}(t, x ; b)-q_{f}(t, x ; a)=q_{f}(t, p ; b)-q_{f}(t, p ; a)-\frac{b-a}{t}(x-p), \quad x \in \mathbb{R}$.

The proof is trivial. 
As a consequence, we have

Lemma 2.5 Let $f \in C_{p}(\mathbb{R})$ and $a, b, p, t \in \mathbb{R}$ with $a<b$ and $t>0$. If $H_{t} f(p)=$ $q_{f}(t, p ; a)=q_{f}(t, p ; b)$, then

$$
\frac{f(b)-f(y)}{b-y}-\frac{f(y)-f(a)}{y-a} \leq-\frac{b-a}{2 t}, \quad y \in(a, b) .
$$

Proof Let $y \in(a, b)$. Since $H_{t} f(p)=q_{f}(t, p ; a)$, we have $q_{f}(t, p ; a) \leq$ $q_{f}(t, p ; y)$. By Lemma 2.4 , we have $q_{f}(t, \cdot ; a) \leq q_{f}(t, \cdot ; y)$ on $(-\infty, p]$. On the other hand, since $H_{t} f(p)=q_{f}(t, p ; b)$, we have $q_{f}(t, p ; b) \leq q_{f}(t, p ; y)$. By Lemma 2.4, we have $q_{f}(t, \cdot ; b) \leq q_{f}(t, \cdot ; y)$ on $[p, \infty)$. Thus, (2.4) holds. We conclude the proof exploiting Lemma 2.3 .

The following lemma will be repeatedly used in the paper. For the proof, see [7, Lemma 4.1, p. 356].

Lemma 2.6 Let $f \in C_{p}(\mathbb{R})$. If $f(z) \geq 0$ for all $z \in \mathbb{R}$, then

$$
H_{t} f(x)=\min _{z \in[0,1]} q_{f}(t, x ; z), \quad(t, x) \in(0, \infty) \times[0,1]
$$

Next lemma will be crucial for proving Theorem 1.5.

Lemma 2.7 Let $\left\{\Gamma_{n}\right\}_{n \in \mathbb{N}_{0}}$ be a sequence of partitions of $[0,1]$ satisfying (D1), (D2), and with $\left|\Gamma_{n}\right| \nrightarrow \rightarrow 0$ as $n \rightarrow \infty$. Then there exist constants $\alpha, \beta \in[0,1]$ with $\alpha<\beta$ such that

$$
(\alpha, \beta) \cap \Gamma=\emptyset,
$$

where $\Gamma=\bigcup_{n=0}^{\infty} \Gamma_{n}$.

Proof By (D2), we have $\left|\Gamma_{n}\right| \geq\left|\Gamma_{n+1}\right|$ for each $n \in \mathbb{N}_{0}$. Thus, $\delta:=\lim _{n \rightarrow \infty}\left|\Gamma_{n}\right|>0$ does exist. We can in addition find sequences $a_{n} \in \Gamma_{n}, b_{n} \in \Gamma_{n}$ converging respectively, up to subsequences, to $\alpha, \beta$ in $[0,1]$ with $|\alpha-\beta|=\delta$.

Now, suppose for purposes of contradiction that $\gamma \in(\alpha, \beta) \cap \Gamma \neq \emptyset$. Given any $\varepsilon>0$, we have $\left|a_{n}-\alpha\right|<\varepsilon,\left|b_{n}-\beta\right|<\varepsilon$ for $n$ large, and consequently

$$
\begin{aligned}
\left|a_{n}-\gamma\right|+\left|b_{n}-\gamma\right| & \leq\left|a_{n}-\alpha\right|+|\alpha-\gamma|+\left|b_{n}-\beta\right|+|\beta-\gamma| \\
& \leq \delta+2 \varepsilon .
\end{aligned}
$$

Taking $\varepsilon<\frac{\delta}{2}$, we see that

$$
\text { either }\left|a_{n}-\gamma\right|<\delta \text { or }\left|b_{n}-\gamma\right|<\delta
$$

which is in contradiction with the very definition of $\delta$. 
For given $a \in[0,1]$ and $s, t \in \mathbb{R}$ with $0<s<t$, let

$$
\rho(x)=\frac{(t-s) a+s x}{t}, \quad x \in[0,1]
$$

Note that $0 \leq \rho(x) \leq 1$ and $\rho$ is strictly increasing on $[0,1]$. Note further that $y=\rho(x)$ is the unique minimizer over $[0,1]$ of the function $F:[0,1] \rightarrow \mathbb{R}$ defined by

$$
F(y)=q_{f}(s, y ; a)+\frac{(x-y)^{2}}{2(t-s)}, \quad y \in[0,1]
$$

and the value of the minimum is $q_{f}(t, x ; a)$.

A relevant fact is:

Lemma 2.8 Let $a \in[0,1]$ and $s, t \in \mathbb{R}$ with $0<s<t$. Let $f \in C_{p}(\mathbb{R})$ be $a$ nonnegative function on $\mathbb{R}$. If there exist constants $\alpha, \beta$ with $0 \leq \alpha<\beta \leq 1$ such that

$$
q_{f}(t, x ; a)=\min _{y \in[0,1]}\left[H_{s} f(y)+\frac{(x-y)^{2}}{2(t-s)}\right], \quad x \in[\alpha, \beta],
$$

then

$$
H_{s} f(y)=q_{f}(s, y ; a), \quad y \in[\rho(\alpha), \rho(\beta)] .
$$

Proof Suppose that (2.9) is false. Then, by the definition of $H_{S} f$ in (1.2), there exist constants $\alpha^{\prime}, \beta^{\prime}$ with $0 \leq \alpha<\alpha^{\prime}<\beta^{\prime}<\beta \leq 1$ such that

$$
H_{s} f(y)<q_{f}(s, y ; a), \quad y \in\left[\rho\left(\alpha^{\prime}\right), \rho\left(\beta^{\prime}\right)\right] .
$$

Take $\varepsilon>0$ so that

$$
H_{s} f(y)+\varepsilon<q_{f}(s, y ; a), \quad y \in\left[\rho\left(\alpha^{\prime}\right), \rho\left(\beta^{\prime}\right)\right] .
$$

Set $J=\left[\rho\left(\alpha^{\prime}\right), \rho\left(\beta^{\prime}\right)\right]$. Then, by (2.8), we have

$$
q_{f}(t, x ; a) \leq \min _{y \in J}\left[H_{s} f(y)+\frac{(x-y)^{2}}{2(t-s)}\right], \quad x \in[\alpha, \beta] .
$$

We choose $\delta>0$ so that $0<\delta<\frac{\beta^{\prime}-\alpha^{\prime}}{2}$. Then $\alpha^{\prime}<\frac{\alpha^{\prime}+\beta^{\prime}}{2}-\delta<\frac{\alpha^{\prime}+\beta^{\prime}}{2}+\delta<\beta^{\prime}$. Let us choose $x$ so that

$$
\frac{\alpha^{\prime}+\beta^{\prime}}{2}-\delta<x<\frac{\alpha^{\prime}+\beta^{\prime}}{2}+\delta .
$$

We note that $\rho(x) \in J$, since $\alpha^{\prime}<x<\beta^{\prime}$. Since $F$ of (2.7) takes its minimum $q_{f}(t, x ; a)$ over $[0,1]$ only at $y=\rho(x)$, we have, by $(2.10)$, 


$$
\begin{aligned}
& \min _{y \in J}\left[H_{S} f(y)+\frac{(x-y)^{2}}{2(t-s)}\right] \\
& \quad \leq \min _{y \in J}\left[q_{f}(s, y ; a)-\varepsilon+\frac{(x-y)^{2}}{2(t-s)}\right]=\min _{y \in J} F(y)-\varepsilon=q_{f}(t, x ; a)-\varepsilon .
\end{aligned}
$$

This is a contradiction by (2.11). Therefore, (2.9) holds true.

\section{The main results}

In this section, we state and prove our main results. We start by Propositions 1.3 and 1.4. A preliminary fact is:

Lemma 3.1 Let $f \in \mathcal{R}$. Then $f(x)>0$ for $x \in(0,1)$.

Proof Let $f \in \mathcal{R}_{\theta}$ for some $\theta \in(0, \infty)$, and $\left\{\Gamma_{n}\right\}$ the admissible sequence of partitions of the interval $[0,1]$ appearing in Definition 1.2. Thus $\Gamma_{0}=\{0,1\}$. By Lemma 2.2 and (R1) with $n=0$, we have $f(x) \geq \theta x(1-x)>0$ for $x \in(0,1)$, since $f(0)=$ $f(1)=0$.

In the following two lemmas, we let $\left\{\Gamma_{n}\right\}$ be the admissible sequence of partitions of the interval $[0,1]$ appearing in Definition 1.2 and we set, for each $n \in \mathbb{N}_{0}$,

$$
\begin{array}{ll}
\alpha_{n}(x)=\max \left\{a_{k}^{(n)} \in \Gamma_{n} \mid a_{k}^{(n)}<x\right\}, & x \in(0,1], \\
\beta_{n}(x)=\min \left\{a_{k}^{(n)} \in \Gamma_{n} \mid x<a_{k}^{(n)}\right\}, & x \in[0,1) .
\end{array}
$$

We set $\alpha_{n}(0)=\alpha_{n}(1)-1$ and $\beta_{n}(1)=\beta_{n}(0)+1$. By (D3), we see that

$$
\lim _{n \rightarrow \infty} \alpha_{n}(x)=\lim _{n \rightarrow \infty} \beta_{n}(x)=x \quad \text { for each } x \in[0,1]
$$

We set

$$
\Gamma=\bigcup_{n=0}^{+\infty} \Gamma_{n} .
$$

It is apparent that $\Gamma$ is a countable and dense set of $[0,1]$.

We break through the proof of Proposition 1.3 by the following two lemmas.

Lemma 3.2 Let $f \in \mathcal{R}$. If $x \in[0,1] \backslash \Gamma$, then $D^{-} f(x)=\emptyset$. Thus $f$ is nondifferentiable at each point of $[0,1] \backslash \Gamma$.

Proof For $x \in[0,1] \backslash \Gamma$, let $\left\{\alpha_{n}(x)\right\}$ and $\left\{\beta_{n}(x)\right\}$ be the sequences defined in (3.1) and (3.2), respectively. 
Let $n \in \mathbb{N}_{0}$. Since $x \in[0,1] \backslash \Gamma$, there exists a unique integer $j \in\left\{0,1,2, \ldots, N_{n}-\right.$ $1\}$ such that $\alpha_{n}(x)=a_{j}^{(n)}$ and $\beta_{n}(x)=a_{j+1}^{(n)}$. Thus, by Definition 1.2, we have

$$
\frac{f\left(\beta_{n}(x)\right)-f(x)}{\beta_{n}(x)-x}-\frac{f(x)-f\left(\alpha_{n}(x)\right)}{x-\alpha_{n}(x)} \leq-\theta
$$

for a suitable $\theta>0$. By (3.3), this implies

$$
\begin{aligned}
\mathfrak{D}_{+} f(x) & \leq \liminf _{n \rightarrow \infty} \frac{f\left(\beta_{n}(x)\right)-f(x)}{\beta_{n}(x)-x} \leq \limsup _{n \rightarrow \infty} \frac{f\left(\alpha_{n}(x)\right)-f(x)}{\alpha_{n}(x)-x}-\theta \\
& \leq \mathfrak{D}^{-} f(x)-\theta .
\end{aligned}
$$

We then invoke Proposition 2.1 to get the assertion.

Lemma 3.3 Let $f \in \mathcal{R}$. If $x_{0} \in \Gamma$, then $D^{-} f\left(x_{0}\right)=\mathbb{R}$. Thus $f$ is non-differentiable at each point of $\Gamma$.

Proof Let $n \in \mathbb{N}_{0}$ and $x_{0} \in \Gamma \cap(0,1)$. By (R1), we have

$$
\frac{f(x)-f\left(x_{0}\right)}{x-x_{0}} \geq \frac{f(x)-f\left(\beta_{n}\left(x_{0}\right)\right)}{x-\beta_{n}\left(x_{0}\right)}+\theta, \quad x \in\left(x_{0}, \beta_{n}\left(x_{0}\right)\right)
$$

for a suitable $\theta>0$. By freezing $n$ and letting $x$ go to $x_{0}$, we get

$$
\mathfrak{D}_{+} f\left(x_{0}\right) \geq \frac{f\left(x_{0}\right)-f\left(\beta_{n}\left(x_{0}\right)\right)}{x_{0}-\beta_{n}\left(x_{0}\right)}+\theta \text { for any } n .
$$

By letting $n$ go to $\infty$, we get, by (3.3),

$$
\liminf _{n \rightarrow \infty} \frac{f\left(x_{0}\right)-f\left(\beta_{n}\left(x_{0}\right)\right)}{x_{0}-\beta_{n}\left(x_{0}\right)} \geq \mathfrak{D}_{+} f\left(x_{0}\right) .
$$

Relations (3.4), (3.5) are compatible if and only if $\mathfrak{D}_{+} f\left(x_{0}\right)=+\infty$. Arguing as above with obvious adaptations we also get $\mathfrak{D}^{-} f\left(x_{0}\right)=-\infty$. Finally, by Proposition 2.1,

$$
D^{-} f\left(x_{0}\right)=\llbracket \mathfrak{D}^{-} f\left(x_{0}\right), \mathfrak{D}_{+} f\left(x_{0}\right) \rrbracket=\mathbb{R},
$$

as was asserted. When $x_{0}=0$ or $x_{0}=1$, we can show that $D^{-} f\left(x_{0}\right)=\mathbb{R}$ similarly, since $f \in C_{p}(\mathbb{R})$.

Remark 3.4 When $f \in C_{p}(\mathbb{R})$ is given by (1.5), Ferrera and Gómez Gil [6] showed that $f$ satisfies

$$
D^{-} f\left(x_{0}\right)=\emptyset \quad\left(x_{0} \in[0,1] \backslash \Gamma\right), \quad D^{-} f\left(x_{0}\right)=\mathbb{R} \quad\left(x_{0} \in \Gamma\right) .
$$

Next, we prove Proposition 1.4. 
Proof of Proposition 1.4 Let $f \in \mathcal{R}$. By Lemmas 3.1 and 2.6, we obtain (2.6).

Choose a constant $\theta \in(0, \infty)$ and an admissible sequence $\left\{\Gamma_{n}\right\}$ of partitions $\Gamma_{n}=\left\{a_{k}^{(n)}\right\}_{k=0}^{N_{n}}$ of the interval [0,1] appearing in Definition 1.2. Now, fix $(n, t) \in$ $\mathbb{N}_{0} \times\left[\frac{\left|\Gamma_{n}\right|}{2 \theta}, \infty\right)$ arbitrarily. For any $y \in\left(a_{k}^{(n)}, a_{k+1}^{(n)}\right)$ and $k \in\left\{0,1,2, \cdots, N_{n}-1\right\}$, we have

$$
\frac{f\left(a_{k+1}^{(n)}\right)-f(y)}{a_{k+1}^{(n)}-y}-\frac{f(y)-f\left(a_{k}^{(n)}\right)}{y-a_{k}^{(n)}} \leq-\theta \leq-\frac{a_{k+1}^{(n)}-a_{k}^{(n)}}{2 t},
$$

since $t \geq \frac{\left|\Gamma_{n}\right|}{2 \theta} \geq \frac{a_{k+1}^{(n)}-a_{k}^{(n)}}{2 \theta}$. By Lemma 2.3 and (2.6), it is easy to see that $\left\{H_{t} f\right\}$ satisfies (HJ).

The proof of Theorem 1.5 passes through the definition of a more general framework. For this purpose, we introduce a new class of functions contained in $C_{p}(\mathbb{R})$.

Definition 3.5 (The class $\mathcal{H}$ ) A function $f \in C_{p}(\mathbb{R})$ is said to belong to $\mathcal{H}$ if there exist an admissible sequence of partitions $\left\{\Gamma_{n}\right\}$ of the interval $[0,1]$ and a positive and bounded sequence $\left\{K_{n}\right\}_{n \in \mathbb{N}_{0}}$ such that $t_{n}:=K_{n}\left|\Gamma_{n}\right|$ satisfies

(H1) $t_{0}>t_{1}>t_{2}>\cdots>t_{n}>t_{n+1} \rightarrow 0$ as $n \rightarrow \infty$.

(H2) For each $n \in \mathbb{N}_{0}$,

$$
H_{t_{n}} f(x)=\min _{y \in \Gamma_{n}} q_{f}\left(t_{n}, x ; y\right), \quad x \in[0,1] .
$$

Proposition 3.6 Let $f \in C_{p}(\mathbb{R})$. If $\left\{H_{t} f\right\}$ satisfies $(\mathrm{HJ})$, then $f \in \mathcal{H}$. In particular, $\mathcal{R} \subset \mathcal{H}$.

Proof Let $f \in C_{p}(\mathbb{R})$ such that $\left\{H_{t} f\right\}$ satisfies $(\mathrm{HJ})$. Choose a constant $\theta \in(0, \infty)$ and the admissible sequence of partitions $\left\{\Gamma_{n}\right\}$ of the interval $[0,1]$ appearing in $(\mathrm{HJ})$.

Set

$$
K_{n}=\frac{1}{2 \theta}+\frac{1}{n+1}, \quad n \in \mathbb{N}_{0} .
$$

Then $\left\{K_{n}\right\}$ is a positive and bounded sequence. Since $\Gamma_{n} \subset \Gamma_{n+1}$ for $n \in \mathbb{N}_{0}$, we have $\left|\Gamma_{n}\right| \geq\left|\Gamma_{n+1}\right|$ for $n \in \mathbb{N}_{0}$. Let $\left\{t_{n}\right\}$ be the sequence in Definition 3.5. For $n \in \mathbb{N}_{0}$, we have

$$
\begin{gathered}
t_{n}-t_{n+1}=\left(\frac{1}{2 \theta}+\frac{1}{n+1}\right)\left|\Gamma_{n}\right|-\left(\frac{1}{2 \theta}+\frac{1}{n+2}\right)\left|\Gamma_{n+1}\right| \\
\geq\left(\frac{1}{2 \theta}+\frac{1}{n+1}\right)\left|\Gamma_{n+1}\right|-\left(\frac{1}{2 \theta}+\frac{1}{n+2}\right)\left|\Gamma_{n+1}\right|=\frac{\left|\Gamma_{n+1}\right|}{(n+1)(n+2)}>0 .
\end{gathered}
$$

Since $\left|\Gamma_{n}\right| \rightarrow 0$ as $n \rightarrow \infty$, we have $t_{n} \rightarrow 0$ as $n \rightarrow \infty$. Hence $\left\{t_{n}\right\}$ satisfies (H1) of Definition 3.5. Since $t_{n}>\frac{\left|\Gamma_{n}\right|}{2 \theta}$ and $\left\{H_{t} f\right\}$ satisfies (HJ), $f$ satisfies (H2) of Definition 3.5. Therefore, $f \in \mathcal{H}$.

When $f \in \mathcal{R}$, we see that $f \in \mathcal{H}$ by Proposition 1.4 and the arguments above. 
Lemma 3.7 Let $f \in \mathcal{H}$. Then $f(x)>0$ for $x \in(0,1)$.

Proof Let $f \in \mathcal{H}$. By (H2) with $n=0$ in Definition 3.5, we have

$$
H_{t_{0}} f(x)=\min \left\{\frac{x^{2}}{2 t_{0}}, \frac{(1-x)^{2}}{2 t_{0}}\right\}>0, \quad x \in(0,1) .
$$

Since $f(x) \geq H_{t_{0}} f(x)$ for $x \in \mathbb{R}$ by (1.2), we conclude the lemma.

In the following, we always consider a function $f \in \mathcal{H}$ and the admissible sequence $\left\{\Gamma_{n}\right\}$ of partitions of $[0,1]$ appearing in Definition 3.5. We set

$$
E_{n}(y)=\left\{x \in[0,1] \mid H_{t_{n}} f(x)=q_{f}\left(t_{n}, x ; y\right)\right\}, \quad n \in \mathbb{N}_{0}, y \in \Gamma_{n},
$$

where $\left\{t_{n}\right\}$ is the sequence appearing in Definition 3.5.

Lemma 3.8 For every $n \in \mathbb{N}_{0}$ and $y \in \Gamma_{n}, E_{n}(y)$ is a closed interval in $[0,1]$, possibly reduced to a singleton or empty.

Let $x, y, z, t \in \mathbb{R}$ with $y \neq z$ and $t>0$. Note that when $z>y$, the inequality $q_{f}(t, x ; z) \geq q_{f}(t, x ; y)$ holds if and only if the inequality

$$
x \leq \frac{y+z}{2}+t \frac{f(z)-f(y)}{z-y}
$$

holds. Note also that when $z<y$, the inequality $q_{f}(t, x ; z) \geq q_{f}(t, x ; y)$ holds if and only if the inequality

$$
x \geq \frac{y+z}{2}+t \frac{f(z)-f(y)}{z-y}
$$

holds.

Proof Let $x, \hat{x} \in E_{n}(y)$ and $\lambda \in(0,1)$. Then, for any $z \in \Gamma_{n} \backslash\{y\}$, we have

$$
q_{f}\left(t_{n}, x ; z\right) \geq q_{f}\left(t_{n}, x ; y\right), \quad q_{f}\left(t_{n}, \hat{x} ; z\right) \geq q_{f}\left(t_{n}, \hat{x} ; y\right) .
$$

When $z>y$, we have, from these inequalities,

$$
x, \hat{x} \leq \frac{y+z}{2}+t_{n} \frac{f(z)-f(y)}{z-y},
$$

so that

$$
\lambda x+(1-\lambda) \hat{x} \leq \frac{y+z}{2}+t_{n} \frac{f(z)-f(y)}{z-y} .
$$


Thus the last inequality implies that

$$
q_{f}\left(t_{n}, \lambda x+(1-\lambda) \hat{x} ; z\right) \geq q_{f}\left(t_{n}, \lambda x+(1-\lambda) \hat{x} ; y\right) .
$$

Similarly, when $z<y$, we have also the inequality (3.7) from (3.6). This implies that $\lambda x+(1-\lambda) \hat{x} \in E_{n}(y)$. Hence $E_{n}(y)$ is a convex set in $\mathbb{R}$. Since $H_{t_{n}} f$ is continuous on $\mathbb{R}, E_{n}(y)$ is a closed set in $\mathbb{R}$, so that it is a closed interval. The proof is complete.

Next, we set

$$
\hat{\Gamma}_{n}=\left\{y \in \Gamma_{n} \mid \text { the interior of } E_{n}(y) \text { is not empty }\right\} \text {. }
$$

By (H2) of Definition 3.5, we see that

$$
H_{t_{n}} f(x)=\min _{y \in \hat{\Gamma}_{n}} q_{f}\left(t_{n}, x ; y\right), \quad n \in \mathbb{N}_{0}, x \in[0,1],
$$

since a point $y \in \Gamma_{n}$ such that $E_{n}(y)$ is the empty set or a singleton never contributes to the minimization of $q_{f}\left(t_{n}, \cdot, y\right)$ over $\Gamma_{n}$. We proceed showing that $\left\{\hat{\Gamma}_{n}\right\}$ is an admissible sequence of partitions of $[0,1]$.

Lemma 3.9 For every $n \in \mathbb{N}_{0}$, $\min \hat{\Gamma}_{n}=0$ and $\max \hat{\Gamma}_{n}=1$. In particular, $\hat{\Gamma}_{0}=$ $\{0,1\}$.

Proof Let $y_{0}:=\min \left\{y \in \Gamma_{n} \mid y>0\right\}$. Then $0<y_{0} \leq 1$. We show that $\left[0, \frac{y_{0}}{2}\right) \subset$ $E_{n}(0)$, which shows that $0 \in \hat{\Gamma}_{n}$ and $\min \hat{\Gamma}_{n}=0$. Indeed, let $y \in \Gamma_{n}$ with $0<y \leq 1$. Since $f \in \mathcal{H}$, we have $f \geq 0$ on $[0,1]$ by Lemma 3.7. Then, for any $x \in\left[0, \frac{y_{0}}{2}\right)$, we have

$$
\frac{y}{2 t_{n}}(2 x-y)<0 \leq f(y)
$$

since $0 \leq 2 x<y_{0} \leq y$. Thus it is easy to see that

$$
\frac{x^{2}}{2 t_{n}}<f(y)+\frac{1}{2 t_{n}}(x-y)^{2} .
$$

Therefore, $q_{f}\left(t_{n}, x ; 0\right)<q_{f}\left(t_{n}, x ; y\right)$ and $H_{t_{n}} f(x)=q_{f}\left(t_{n}, x ; 0\right)$. This implies that $x \in E_{n}(0)$, so that $\left[0, \frac{y_{0}}{2}\right) \subset E_{n}(0)$ as was desired.

Next, let $y_{1}:=\max \left\{y \in \Gamma_{n} \mid y<1\right\}$. Then $0 \leq y_{1}<1$. Similarly to the proof above, we can show that $\left(\frac{y_{1}+1}{2}, 1\right] \subset E_{n}(1)$, which shows that $1 \in \hat{\Gamma}_{n}$ and $\max \hat{\Gamma}_{n}=1$.

When $n=0$, we have $\hat{\Gamma}_{0} \subset \Gamma_{0}=\{0,1\}$. Since $\min \hat{\Gamma}_{0}=0$ and $\max \hat{\Gamma}_{0}=1$, we have $\hat{\Gamma}_{0}=\{0,1\}$. The proof is complete.

Lemma 3.10 For every $n \in \mathbb{N}_{0}, \hat{\Gamma}_{n} \subset \hat{\Gamma}_{n+1}$. 
Proof Since $f \in \mathcal{H}$, we have $f \geq 0$ on $[0,1]$ by Lemma 3.7. For $n \in \mathbb{N}_{0}$, let $s=t_{n+1}$ and $t=t_{n}$, where $\left\{t_{n}\right\}$ is the sequence in Definition 3.5. By (H1) of Definition 3.5, we have $0<s<t$. Then using the semigroup property $H_{t} f=H_{t-s} \circ H_{s} f$ and Lemma 2.8, we see easily that if $a \in \hat{\Gamma}_{n}$ then $a \in \hat{\Gamma}_{n+1}$. The proof is complete.

Lemma $3.11\left|\hat{\Gamma}_{n}\right| \rightarrow 0$ as $n \rightarrow \infty$.

Proof Since $\hat{\Gamma}_{n} \subset \Gamma_{n}$ for each $n \in \mathbb{N}_{0}, \hat{\Gamma}_{n}$ is a finite set. By Lemmas 3.9 and 3.10, $\left\{\hat{\Gamma}_{n}\right\}_{n \in \mathbb{N}_{0}}$ satisfies (D1) and (D2) of Definition 1.1 of Sect. 1 where $\Gamma_{n}$ is replaced by $\hat{\Gamma}_{n}$. Suppose that the conclusion of the theorem is false. Then, by Lemma 2.7 , there exist constants $\alpha, \beta \in[0,1]$ with $\alpha<\beta$ such that

$$
(\alpha, \beta) \cap \hat{\Gamma}=\emptyset,
$$

where $\hat{\Gamma}=\bigcup_{n=0}^{\infty} \hat{\Gamma}_{n}$.

Choose $\delta \in\left(0, \frac{\beta-\alpha}{2}\right)$, and fix $x \in(\alpha+\delta, \beta-\delta)$ arbitrarily. Let $n \in \mathbb{N}_{0}$ and $y \in \hat{\Gamma}_{n}$. Then $y \leq \alpha$ or $\beta \leq y$. When $y \leq \alpha$, we have $x-y \geq \alpha+\delta-\alpha=\delta$, so that $(x-y)^{2} \geq \delta^{2}$. When $\beta \leq y$, we have $y-x \geq \beta-(\beta-\delta)=\delta$, so that $(x-y)^{2} \geq \delta^{2}$. Thus, for any $y \in \hat{\Gamma}_{n}$, we have $(x-y)^{2} \geq \overline{\delta^{2}}$.

Let $\left\{t_{n}\right\}$ be the sequence in Definition 3.5. Then $t_{n} \rightarrow 0$ as $n \rightarrow \infty$. Note that $f \geq 0$ on $[0,1]$ by Lemma 3.7 , since $f \in \mathcal{H}$. Then

$$
q_{f}\left(t_{n}, x ; y\right)=f(y)+\frac{(x-y)^{2}}{2 t_{n}} \geq \frac{\delta^{2}}{2 t_{n}}, \quad x \in(\alpha+\delta, \beta-\delta), n \in \mathbb{N}_{0}, \quad y \in \hat{\Gamma}_{n} .
$$

Thus

$$
\min _{y \in \hat{\Gamma}_{n}} q_{f}\left(t_{n}, x ; y\right) \geq \frac{\delta^{2}}{2 t_{n}}, \quad x \in(\alpha+\delta, \beta-\delta), n \in \mathbb{N}_{0} .
$$

By (3.8), we have $H_{t_{n}} f(x) \rightarrow \infty$ as $n \rightarrow \infty$ for $x \in(\alpha+\delta, \beta-\delta)$. However, $\left\{H_{t_{n}} f\right\}$ must converges to $f$ as $n \rightarrow \infty$ uniformly on $\mathbb{R}$. This is a contradiction, and the conclusion holds true. The proof is complete.

From Lemmas 3.9, 3.10 and 3.11, we have

Proposition $3.12\left\{\hat{\Gamma}_{n}\right\}$ is an admissible sequence of partitions of the interval $[0,1]$ with $\hat{\Gamma}_{n} \subset \Gamma_{n}$ for all $n \in \mathbb{N}_{0}$.

Remark 3.13 We do not know whether $\hat{\Gamma}_{n}=\Gamma_{n}$ for each $n \in \mathbb{N}_{0}$.

Now, we set

$$
\begin{aligned}
& \left.N_{n}=\text { (the number of elements of } \hat{\Gamma}_{n}\right)-1, \\
& \hat{\Gamma}_{n}=\left\{a_{0}^{(n)}, a_{1}^{(n)}, a_{2}^{(n)}, \cdots, a_{N_{n}}^{(n)}\right\} \text { with } 0=a_{0}^{(n)}<a_{1}^{(n)}<a_{2}^{(n)} \cdots<a_{N_{n}}^{(n)}=1 .
\end{aligned}
$$


In addition, we set

$$
p_{0}^{(n)}=0, \quad p_{j}^{(n)}=x_{a_{j-1}^{(n)}, a_{j}^{(n)}}\left(t_{n}\right), \quad j \in\left\{1,2, \ldots, N_{n}\right\},
$$

where $x_{a, b}(t)$ is defined by (2.3) for given $a, b, t \in \mathbb{R}$ with $a<b$ and $t>0$.

Lemma 3.14 For every $n \in \mathbb{N}_{0}$, we have

$$
H_{t_{n}} f\left(p_{j}^{(n)}\right)=q_{f}\left(t_{n}, p_{j}^{(n)} ; a_{j}^{(n)}\right)=q_{f}\left(t_{n}, p_{j}^{(n)} ; a_{j-1}^{(n)}\right), \quad j \in\left\{1,2, \cdots, N_{n}\right\} .
$$

Proof Fix $n \in \mathbb{N}_{0}$ and let $j \in\left\{1,2, \cdots, N_{n}\right\}$. By the definition of $p_{j}^{(n)}$, we have $q_{f}\left(t_{n}, p_{j}^{(n)} ; a_{j}^{(n)}\right)=q_{f}\left(t_{n}, p_{j}^{(n)} ; a_{j-1}^{(n)}\right)$. Supposing that

$$
H_{t_{n}} f\left(p_{j}^{(n)}\right)<q_{f}\left(t_{n}, p_{j}^{(n)} ; a_{j}^{(n)}\right)=q_{f}\left(t_{n}, p_{j}^{(n)} ; a_{j-1}^{(n)}\right),
$$

we derive a contradiction. In this case, we find a point $c \in \hat{\Gamma}_{n} \backslash\left\{a_{j-1}^{(n)}, a_{j}^{(n)}\right\}$ such that $H_{t_{n}} f\left(p_{j}^{(n)}\right)=q_{f}\left(t_{n}, p_{j}^{(n)} ; c\right)$ by (3.8). Note that $c<a_{j-1}^{(n)}$ or $a_{j}^{(n)}<c$.

First we consider the case $c<a_{j-1}^{(n)}$. By Lemma 2.4, we have

$$
\begin{aligned}
& q_{f}\left(t_{n}, x ; a_{j-1}^{(n)}\right)-q_{f}\left(t_{n}, x ; c\right) \\
& \quad=q_{f}\left(t_{n}, p_{j}^{(n)} ; a_{j-1}^{(n)}\right)-q_{f}\left(t_{n}, p_{j}^{(n)} ; c\right)-\frac{a_{j-1}^{(n)}-c}{t_{n}}\left(x-p_{j}^{(n)}\right) \\
& \quad>-\frac{a_{j-1}^{(n)}-c}{t_{n}}\left(x-p_{j}^{(n)}\right) \geq 0, \quad x \leq p_{j}^{(n)} .
\end{aligned}
$$

On the other hand, by Lemma 2.4 again, we have

$$
\begin{aligned}
q_{f} & \left(t_{n}, x ; a_{j}^{(n)}\right)-q_{f}\left(t_{n}, x ; a_{j-1}^{(n)}\right) \\
& =q_{f}\left(t_{n}, p_{j}^{(n)} ; a_{j}^{(n)}\right)-q_{f}\left(t_{n}, p_{j}^{(n)} ; a_{j-1}^{(n)}\right)-\frac{a_{j}^{(n)}-a_{j-1}^{(n)}}{t_{n}}\left(x-p_{j}^{(n)}\right) \\
& =-\frac{a_{j}^{(n)}-a_{j-1}^{(n)}}{t_{n}}\left(x-p_{j}^{(n)}\right)<0, \quad x>p_{j}^{(n)} .
\end{aligned}
$$

Thus,

$$
q_{f}\left(t_{n}, x ; a_{j-1}^{(n)}\right)>\min \left\{q_{f}\left(t_{n}, x ; c\right), q_{f}\left(t_{n}, x ; a_{j}^{(n)}\right)\right\}, \quad x \in \mathbb{R} .
$$

This implies that $E_{n}\left(a_{j-1}^{(n)}\right)=\emptyset$ and $a_{j-1}^{(n)} \notin \hat{\Gamma}_{n}$. This is a contradiction.

Next we consider the case $a_{j}^{(n)}<c$. Similarly to the arguments above, we can show that $E_{n}\left(a_{j}^{(n)}\right)=\emptyset$ and $a_{j}^{(n)} \notin \hat{\Gamma}_{n}$. This is also a contradiction. 


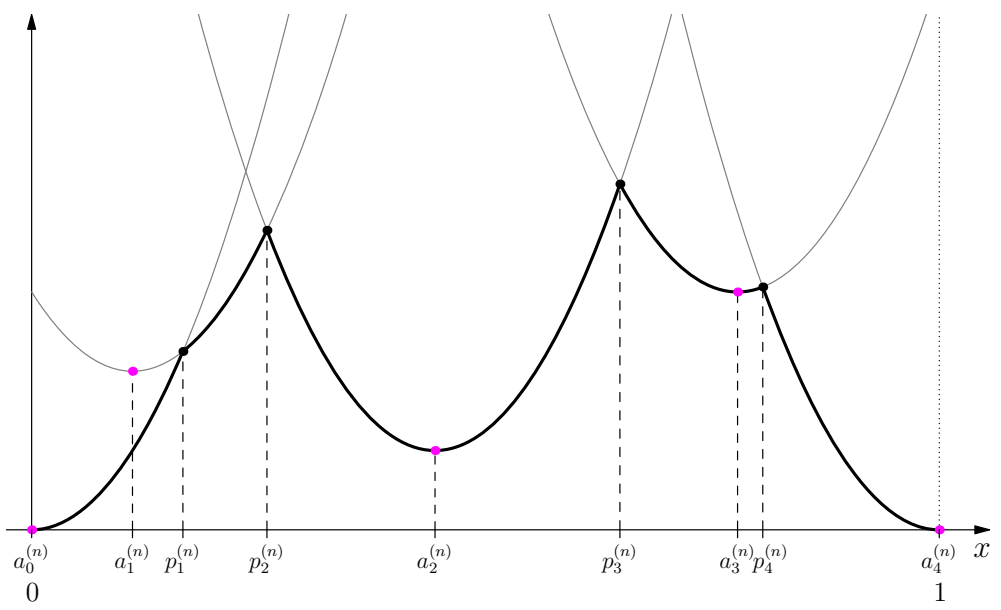

Fig. 1 The graph of $H_{t_{n}} f$ with $N_{n}=4$

Hence we conclude that (3.11) leads to a contradiction. Therefore (3.10) holds true. The proof is complete.

Remark 3.15 Let $n \in \mathbb{N}_{0}$. By Lemma 3.14, we can show that, for each $j \in$ $\left\{0,1,2, \ldots, N_{n}\right\}$,

$$
H_{t_{n}} f=q_{f}\left(t_{n}, \cdot ; a_{j}^{(n)}\right) \quad \text { on }\left[p_{j}^{(n)}, p_{j+1}^{(n)}\right]
$$

where we put $p_{N_{n}+1}^{(n)}=1$ (see Fig. 1). However we do not use this expression in the following. So we omit its proof.

The next result is a partial converse of Proposition 3.6, where condition (A1) of Sect. 1 plays a key role. In Sect. 4, we study examples of admissible sequences of partitions of $[0,1]$ satisfying (A1) or not satisfying (A1).

Theorem 3.16 Let $f \in \mathcal{H}$. Assume that the admissible sequence of partitions $\left\{\Gamma_{n}\right\}$ of the interval $[0,1]$ appearing in the definition of $f \in \mathcal{H}$ satisfies (A1). Then $f \in \mathcal{R}$.

Proof Fix $n \in \mathbb{N}_{0}$. Let $t_{n}=K_{n}\left|\Gamma_{n}\right|$ for $n \in \mathbb{N}_{0}$ and set $M=\sup \left\{K_{n} \mid n \in \mathbb{N}_{0}\right\}$ which appear in Definition 3.5 by the fact that $f \in \mathcal{H}$.

Let $\left\{\hat{\Gamma}_{n}\right\}$ be the admissible sequence of partitions of the interval $[0,1]$ in (3.9). Let $j \in\left\{1,2, \cdots, N_{n}\right\}$. By Lemmas 2.5 and 3.14, we have

$$
\frac{f\left(a_{j}^{(n)}\right)-f(y)}{a_{j}^{(n)}-y}-\frac{f(y)-f\left(a_{j-1}^{(n)}\right)}{y-a_{j-1}^{(n)}} \leq-\frac{a_{j}^{(n)}-a_{j-1}^{(n)}}{2 t_{n}}, \quad y \in\left(a_{j-1}^{(n)}, a_{j}^{(n)}\right) .
$$


Since $\hat{\Gamma}_{n} \subset \Gamma_{n}$ and $\left\{\Gamma_{n}\right\}$ satisfies (A1), we have

$$
a_{j}^{(n)}-a_{j-1}^{(n)} \geq \min \left\{|x-y| \mid x, y \in \hat{\Gamma}_{n}, x \neq y\right\} \geq \min \left\{|x-y| \mid x, y \in \Gamma_{n}, x \neq y\right\} \geq \delta\left|\Gamma_{n}\right| .
$$

Then we have

$$
\frac{f\left(a_{j}^{(n)}\right)-f(y)}{a_{j}^{(n)}-y}-\frac{f(y)-f\left(a_{j-1}^{(n)}\right)}{y-a_{j-1}^{(n)}} \leq-\frac{\delta\left|\Gamma_{n}\right|}{2 K_{n}\left|\Gamma_{n}\right|} \leq-\frac{\delta}{2 M}, \quad y \in\left(a_{j-1}^{(n)}, a_{j}^{(n)}\right) .
$$

Thus $f \in \mathcal{R}$ by Definition 1.2 where $\left\{\Gamma_{n}\right\}$ and $\theta$ are, respectively, replaced by $\left\{\hat{\Gamma}_{n}\right\}$ and $\frac{\delta}{2 M}$. Hence, we conclude the proof.

Remark 3.17 In the proof of Theorem 3.16, the condition (A1) is unavoidable to show that $\mathcal{H} \subset \mathcal{R}$. We do not know whether it is actually necessary to prove that $\mathcal{H} \subset \mathcal{R}$.

Proof of Theorem 1.5 Let $f \in C_{p}(\mathbb{R})$. Since $\left\{H_{t} f\right\}$ satisfies $(\mathrm{HJ})$, we have $f \in \mathcal{H}$ by Proposition 3.6. Here, the admissible sequence $\left\{\Gamma_{n}\right\}$ of partitions of $[0,1]$ appearing in $(\mathrm{HJ})$ gives an admissible sequence of partitions of $[0,1]$ satisfying Definition 3.5. Since $\left\{\Gamma_{n}\right\}$ satisfies (A1), we conclude that $f \in \mathcal{R}$ by Theorem 3.16. By Proposition $1.3, f$ is nowhere differentiable on $\mathbb{R}$.

\section{Examples}

In this section, we give examples of admissible sequences of partitions of the interval $[0,1]$ satisfying (A1) or not satisfying (A1). We also systematically construct examples of functions belonging to the class $\mathcal{R}$.

First we consider (A1). As explained in Sect. 1, $\left\{\Gamma_{n}\right\}$ of (1.1) satisfies (A1) with $\delta=1$. We give one more example of admissible sequences of partitions of the interval $[0,1]$ satisfying (A1) with $\delta=1$.

\section{Example 4.1 Let}

$$
\Gamma_{n}=\left\{\frac{k}{((n+1) !)^{r}} \mid k \in \mathbb{N}_{0}, 0 \leq k \leq((n+1) !)^{r}\right\}, \quad n \in \mathbb{N}_{0},
$$

where $r$ is a positive integer. It is easy to see that $\left\{\Gamma_{n}\right\}$ is an admissible sequence of partitions of the interval $[0,1]$ satisfying $(\mathrm{A} 1)$ with $\delta=1$.

Next, for a given admissible sequence of partitions of the interval $[0,1]$ satisfying (A1), we give a method to construct another admissible sequences of partitions of the interval $[0,1]$ satisfying (A1).

Theorem 4.2 Let $\left\{\Gamma_{n}\right\}$ be an admissible sequence of partitions of the interval $[0,1]$ satisfying (A1) with a constant $\delta \in(0,1]$. Assume that $\varphi$ is a strictly increasing 
function on $[0,1]$ with $\varphi(0)=0$ and $\varphi(1)=1$ such that there exist constant $\alpha, \beta$ $(0<\alpha \leq 1 \leq \beta)$ satisfying

$$
\alpha(x-y) \leq \varphi(x)-\varphi(y) \leq \beta(x-y), \quad 0 \leq y<x \leq 1 .
$$

Then $\left\{\varphi\left(\Gamma_{n}\right)\right\}$ is an admissible sequence of partitions of the interval $[0,1]$ satisfying (A1) with a constant $\delta_{\varphi} \in\left(0, \frac{\alpha \delta}{\beta}\right]$.

Since the proof is clear, we omit it. We give an example of Theorem 4.2.

Example 4.3 For a given $\hat{\delta} \in(0,1]$, let

$$
a=\frac{2 \hat{\delta}}{1+\hat{\delta}} \in(0,1] .
$$

Define the function $\varphi:[0,1] \rightarrow[0,1]$ by

$$
\varphi(t)= \begin{cases}a t, & t \in\left[0, \frac{1}{2}\right], \\ (2-a) t+a-1, & t \in\left(\frac{1}{2}, 1\right] .\end{cases}
$$

Then $\varphi$ satisfies the assumptions of Theorem 4.2 with $\alpha=a$ and $\beta=2-a$, since $a \leq 2-a$. Let

$$
\Gamma_{n}=\left\{\frac{k}{2^{n}} \mid k \in \mathbb{N}_{0}, 0 \leq k \leq 2^{n}\right\}, \quad n \in \mathbb{N}_{0} .
$$

Recall that $\left\{\Gamma_{n}\right\}$ is an admissible sequence of partitions of the interval $[0,1]$ satisfying (A1) with $\delta=1$. Thus, by Theorem 4.2 , we see that $\left\{\varphi\left(\Gamma_{n}\right)\right\}$ is an admissible sequence of partitions of the interval $[0,1]$ satisfying (A1) with a constant $\delta_{\varphi} \in(0, \hat{\delta}]$, since

$$
\frac{\alpha \delta}{\beta}=\frac{a \cdot 1}{2-a}=\hat{\delta}
$$

Finally, we give an example of admissible sequences of partitions of the interval $[0,1]$ not satisfying (A1).

Example 4.4 Let $\Gamma_{0}=\{0,1\}$, and

$$
\begin{aligned}
\Gamma_{n}=\left\{\frac{k}{2^{n}} \mid\right. & \left.k \in \mathbb{N}_{0}, 0 \leq k \leq 2^{n-1}\right\} \\
& \cup\left\{\frac{\ell}{2^{2 n}} \mid \ell \in \mathbb{N}_{0}, 2^{n-1}+1 \leq \ell \leq 2^{2 n}\right\}
\end{aligned}
$$

for a positive integer $n$. It is easy to see that $\left\{\Gamma_{n}\right\}$ is an admissible sequence of partitions of the interval $[0,1]$ such that

$$
\left|\Gamma_{n}\right|=\frac{1}{2^{n}}, \quad n \in \mathbb{N}_{0} .
$$


Since

$$
\min \left\{|x-y| \mid x, y \in \Gamma_{n}, x \neq y\right\}=\frac{1}{2^{2 n}}, \quad n \in \mathbb{N}_{0},
$$

there exists no $\delta \in(0,1]$ such that $\left\{\Gamma_{n}\right\}$ satisfies (A1).

Next, we systematically construct examples of functions belonging to the class $\mathcal{R}$. Let $\Gamma=\left\{\Gamma_{n}\right\}$ be a given admissible sequence of partitions of the interval $[0,1]$. Here we do not necessarily assume that $\left\{\Gamma_{n}\right\}$ satisfies (A1). We show that all the functions of the form

$$
T_{\Gamma, \Psi}(x)=\sum_{j=0}^{\infty}\left|\Gamma_{j}\right| \psi_{j}\left(\left|\Gamma_{j}\right|^{-1} d\left(x, \Gamma_{j}\right)\right), \quad x \in[0,1],
$$

belong to $\mathcal{R}$, provided that $\sum_{j=0}^{\infty}\left|\Gamma_{j}\right|<+\infty$, where $\Psi=\left\{\psi_{j}\right\}$ is a sequence of functions satisfying:

(A2) For each $j \in \mathbb{N}_{0}, \psi_{j}$ is concave and strictly increasing on $\left[0, \frac{1}{2}\right]$ with $\psi_{j}(0)=0$ such that

$$
K:=\inf _{j \in \mathbb{N}_{0}} \psi_{j}\left(\frac{1}{2}\right)>0, \quad L:=\sup _{j \in \mathbb{N}_{0}} \psi_{j}\left(\frac{1}{2}\right)<\infty .
$$

Note that the choice of $\psi_{j}(x)=x$ for all $j$ is admissible, and in this case $T_{\Gamma, \Psi}$ coincides with the function in (1.5). In this sense, our results includes those of [6] for a function on $\mathbb{R}$ (see [6] and compare to Lemmas 3.2, 3.3 and Remark 3.4).

We provide examples of $\left\{\psi_{j}\right\}$ satisfying (A2).

Example 4.5 For each $j \in \mathbb{N}_{0}$, let $\psi_{j}(x)=A_{j} x^{\alpha_{j}}$ for $x \in\left[0, \frac{1}{2}\right]$, where $\left\{A_{j}\right\}_{j \in \mathbb{N}_{0}}$ and $\left\{\alpha_{j}\right\}_{j \in \mathbb{N}_{0}}$ are sequences satisfying

$$
0<\inf _{j \in \mathbb{N}_{0}} A_{j} \leq \sup _{j \in \mathbb{N}_{0}} A_{j}<+\infty, \quad 0<\alpha_{j} \leq 1\left(j \in \mathbb{N}_{0}\right)
$$

Since $\frac{1}{2} \leq\left(\frac{1}{2}\right)^{\alpha_{j}}<1\left(j \in \mathbb{N}_{0}\right),\left\{\psi_{j}\right\}$ satisfies (A2). In particular, the choice of $\psi_{j}(x)=x$ for all $j$ is admissible.

Under (A2), since $\left|\Gamma_{j}\right|^{-1} d\left(x, \Gamma_{j}\right) \leq \frac{1}{2}$, we have $0 \leq T_{\Gamma, \Psi}(x) \leq L \sum_{j=0}^{\infty}\left|\Gamma_{j}\right|$, so that if $\sum_{j=0}^{\infty}\left|\Gamma_{j}\right|<+\infty$, then $T_{\Gamma, \Psi}$ can be extended as a function of $C_{p}(\mathbb{R})$.

Theorem 4.6 Let $\left\{\Gamma_{n}\right\}$ be an admissible sequence of partitions of the interval $[0,1]$ with $\sum_{n=0}^{\infty}\left|\Gamma_{n}\right|<+\infty$. Assume that (A2) holds. Then the function $T_{\Gamma, \Psi}$ defined by (4.1) belongs to $\mathcal{R}_{K}$, where $K$ is the constant of (4.2).

In order to prove Theorem 4.6, we preliminarily give three lemmas.

Lemma 4.7 Assume (A2). Then we have the inequality

$$
K y \leq \psi_{j}(y), \quad j \in \mathbb{N}_{0}, y \in\left[0, \frac{1}{2}\right]
$$


Proof Let $j \in \mathbb{N}_{0}$ and $y \in\left[0, \frac{1}{2}\right]$. Since $\psi_{j}$ is concave and strictly increasing on $\left[0, \frac{1}{2}\right]$, we derive

$$
\psi_{j}(y) \geq \psi_{j}\left(\frac{y}{2}\right)=\psi_{j}\left((1-y) 0+y \cdot \frac{1}{2}\right) \geq(1-y) \psi_{j}(0)+y \psi_{j}\left(\frac{1}{2}\right)=y \psi_{j}\left(\frac{1}{2}\right) \geq K y .
$$

The proof is complete.

Recall that $d$ is the function defined in (1.4).

Lemma 4.8 Let $n$ be a positive integer and $a, b \in \Gamma_{n}$ with $a<b$ and $(a, b) \cap \Gamma_{n}=\emptyset$. Then, for any $j \in\{0,1,2, \ldots, n-1\}$, we have

$$
\frac{b-x}{b-a} d\left(a, \Gamma_{j}\right)+\frac{x-a}{b-a} d\left(b, \Gamma_{j}\right) \leq d\left(x, \Gamma_{j}\right), \quad x \in(a, b) .
$$

Proof Fix $j \in\{0,1,2, \ldots, n-1\}$ arbitrarily. Then the lemma is an immediate consequence of the fact that $d\left(\cdot, \Gamma_{j}\right)$ is concave on $[a, b]$ since $(a, b) \cap \Gamma_{n}=\emptyset$.

Lemma 4.9 Let $n \in \mathbb{N}_{0}$ and $a, b \in \Gamma_{n}$ with $a<b$ and $(a, b) \cap \Gamma_{n}=\emptyset$. Then, we have

$$
d\left(x, \Gamma_{n}\right) \geq \frac{(x-a)(b-x)}{b-a}, \quad x \in(a, b)
$$

Proof The proof is immediate.

Now, we prove Theorem 4.6.

Proof of Theorem 4.6 To ease notations we will write $T$ in place of $T_{\Gamma, \Psi}$.

In the following, let $n \in \mathbb{N}_{0}$ and $a, b \in \Gamma_{n}$ with $a<b$ and $(a, b) \cap \Gamma_{n}=\emptyset$. By Lemma 2.2, it is sufficient to show the inequality

$$
T(x) \geq \frac{K}{b-a}(x-a)(b-x)+\frac{1}{b-a}[(b-x) T(a)+(x-a) T(b)], x \in(a, b) .
$$

Let first $n=0$. In this case, $\Gamma_{0}=\{0,1\}$. We see that the constants $a, b \in \Gamma_{0}$ with $a<b$ and $(a, b) \cap \Gamma_{0}=\emptyset$ must be $a=0$ and $b=1$. By Lemma 4.9, we have $d\left(x, \Gamma_{0}\right) \geq x(1-x)$ for $x \in(0,1)$. Note that $\psi_{j} \geq 0$ on $\left[0, \frac{1}{2}\right]$ for each $j$ by Lemma 4.7. Since $T(0)=T(1)=0$, we have, by Lemma 4.7, 


$$
\begin{aligned}
T(x)= & \sum_{j=0}^{\infty}\left|\Gamma_{j}\right| \psi_{j}\left(\left|\Gamma_{j}\right|^{-1} d\left(x, \Gamma_{j}\right)\right) \geq\left|\Gamma_{0}\right| \psi_{0}\left(\left|\Gamma_{0}\right|^{-1} d\left(x, \Gamma_{0}\right)\right) \\
& \geq K d\left(x, \Gamma_{0}\right) \geq K x(1-x) \\
& =K x(1-x)+\frac{1}{1-0}[(1-x) T(0)+(x-0) T(1)], \quad x \in(0,1) .
\end{aligned}
$$

This shows (4.3) for $n=0$.

Next, fix a positive integer $n$ arbitrarily. Since $\psi_{j} \geq 0$ on $\left[0, \frac{1}{2}\right]$ for each $j$ by Lemma 4.7, we have

$$
\begin{aligned}
T(x) & =\sum_{j=0}^{\infty}\left|\Gamma_{j}\right| \psi_{j}\left(\left|\Gamma_{j}\right|^{-1} d\left(x, \Gamma_{j}\right)\right) \\
& \geq \sum_{j=0}^{n-1}\left|\Gamma_{j}\right| \psi_{j}\left(\left|\Gamma_{j}\right|^{-1} d\left(x, \Gamma_{j}\right)\right)+\left|\Gamma_{n}\right| \psi_{n}\left(\left|\Gamma_{n}\right|^{-1} d\left(x, \Gamma_{n}\right)\right) \\
& =: I_{n}+J_{n}, x \in(a, b) .
\end{aligned}
$$

Since $\psi_{j}$ is strictly increasing on $\left[0, \frac{1}{2}\right]$, we have, by Lemmas 4.7 and 4.9,

$$
J_{n}=\left|\Gamma_{n}\right| \psi_{n}\left(\left|\Gamma_{n}\right|^{-1} d\left(x, \Gamma_{n}\right)\right) \geq K d\left(x, \Gamma_{n}\right) \geq K \frac{(x-a)(b-x)}{b-a}, \quad x \in(a, b) .
$$

On the other hand, let $j \in\{0,1,2, \cdots, n-1\}$. We have, by Lemma 4.8 and the concavity of $\psi_{j}$ on $\left[0, \frac{1}{2}\right]$,

$$
\begin{aligned}
& \frac{b-x}{b-a} \psi_{j}\left(\left|\Gamma_{j}\right|^{-1} d\left(a, \Gamma_{j}\right)\right)+\frac{x-a}{b-a} \psi_{j}\left(\left|\Gamma_{j}\right|^{-1} d\left(b, \Gamma_{j}\right)\right) \\
& \leq \psi_{j}\left(\frac{b-x}{b-a}\left|\Gamma_{j}\right|^{-1} d\left(a, \Gamma_{j}\right)+\frac{x-a}{b-a}\left|\Gamma_{j}\right|^{-1} d\left(b, \Gamma_{j}\right)\right) \\
& \leq \psi_{j}\left(\left|\Gamma_{j}\right|^{-1} d\left(x, \Gamma_{j}\right)\right), \quad x \in(a, b),
\end{aligned}
$$

since $\psi_{j}$ is strictly increasing on $\left[0, \frac{1}{2}\right]$. Since $a, b \in \Gamma_{n}$, we see that $d\left(a, \Gamma_{j}\right)=$ $d\left(b, \Gamma_{j}\right)=0$ for each $j \in\{n, n+1, \cdots\}$. Hence, by (4.4), we have

$$
\begin{aligned}
& \frac{1}{b-a}[(b-x) T(a)+(x-a) T(b)] \\
= & \frac{b-x}{b-a} \sum_{j=0}^{n-1}\left|\Gamma_{j}\right| \psi_{j}\left(\left|\Gamma_{j}\right|^{-1} d\left(a, \Gamma_{j}\right)\right)+\frac{x-a}{b-a} \sum_{j=0}^{n-1}\left|\Gamma_{j}\right| \psi_{j}\left(\left|\Gamma_{j}\right|^{-1} d\left(b, \Gamma_{j}\right)\right)
\end{aligned}
$$




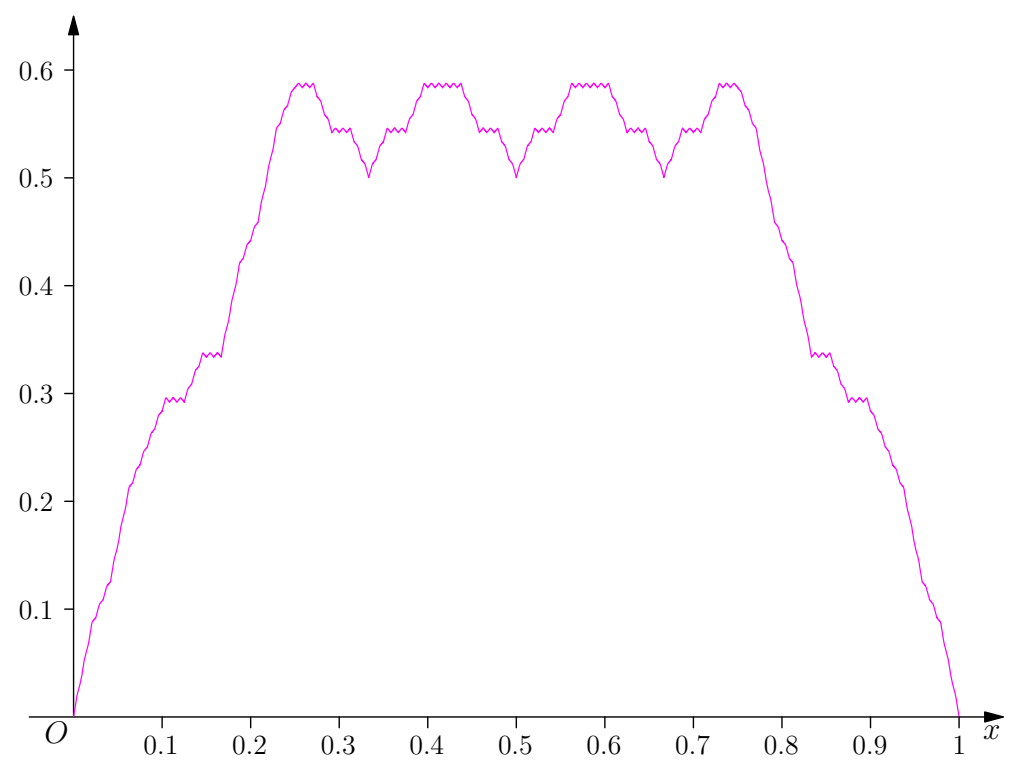

Fig. 2 The grapf of $T_{\Gamma, \Psi}$ when $\psi_{j}(x)=x$

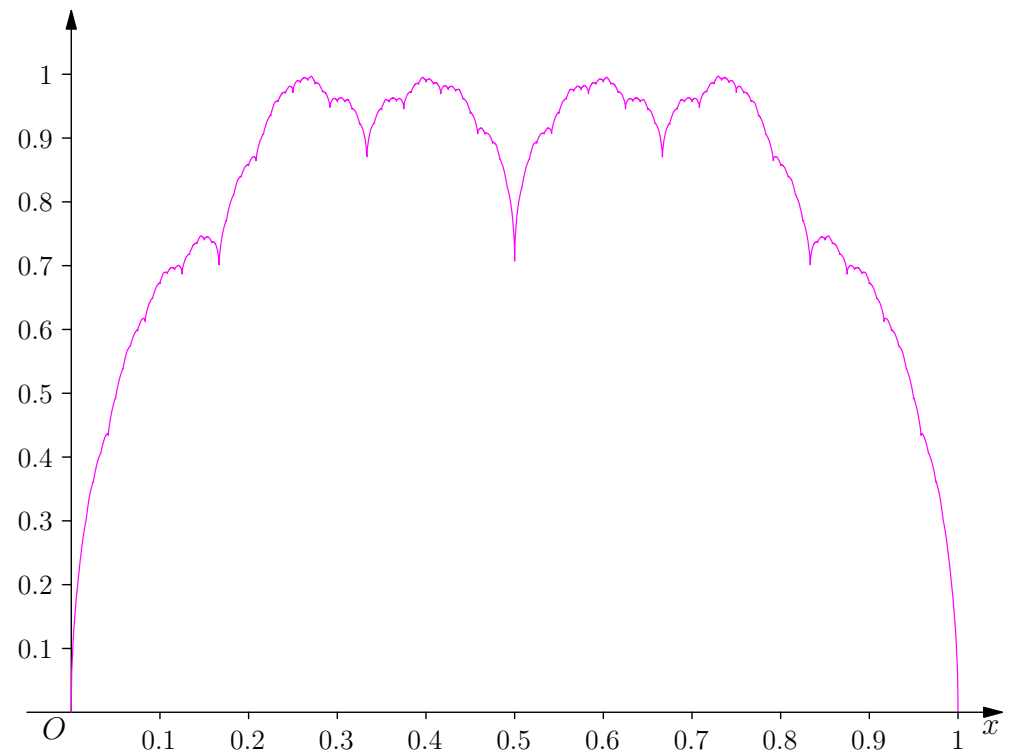

Fig. 3 The grapf of $T_{\Gamma, \Psi}$ when $\psi_{j}(x)=\sqrt{x}$ 


$$
\begin{aligned}
& =\sum_{j=0}^{n-1}\left|\Gamma_{j}\right|\left(\frac{b-x}{b-a} \psi_{j}\left(\left|\Gamma_{j}\right|^{-1} d\left(a, \Gamma_{j}\right)\right)+\frac{x-a}{b-a} \psi_{j}\left(\left|\Gamma_{j}\right|^{-1} d\left(b, \Gamma_{j}\right)\right)\right) \\
& \leq \sum_{j=0}^{n-1}\left|\Gamma_{j}\right| \psi_{j}\left(\left|\Gamma_{j}\right|^{-1} d\left(x, \Gamma_{j}\right)\right)=I_{n}, \quad x \in(a, b) .
\end{aligned}
$$

Therefore, we conclude (4.3). The proof is complete.

Finally we give two figures of functions in $\mathcal{R}$ constructed by Theorem 4.6. Let $\left\{\Gamma_{n}\right\}$ be the admissible sequence of partitions of the interval $[0,1]$ satisfying (A1) given in Example 4.1 with $r=1$. Figure 2 is the grapf of $T_{\Gamma, \Psi}$ of (4.1) when $\psi_{j}(x)=x$ for all $j$. Figure 3 is the grapf of $T_{\Gamma, \Psi}$ of (4.1) when $\psi_{j}(x)=\sqrt{x}$ for all $j$.

Acknowledgements The authors would like to thank the referees for valuable comments.

Open Access This article is licensed under a Creative Commons Attribution 4.0 International License, which permits use, sharing, adaptation, distribution and reproduction in any medium or format, as long as you give appropriate credit to the original author(s) and the source, provide a link to the Creative Commons licence, and indicate if changes were made. The images or other third party material in this article are included in the article's Creative Commons licence, unless indicated otherwise in a credit line to the material. If material is not included in the article's Creative Commons licence and your intended use is not permitted by statutory regulation or exceeds the permitted use, you will need to obtain permission directly from the copyright holder. To view a copy of this licence, visit http://creativecommons.org/licenses/by/4.0/.

\section{References}

1. Allaart, P., Kawamura, K.: The Takagi function: a survey. Real Anal. Exchange 37, 1-54 (2012)

2. Bardi, M., Capuzzo-Dolcetta, I.: Optimal control and viscosity solutions of Hamilton-Jacobi-Bellman equations, systems and control: foundations and applications. Birkhäuser Boston Inc., Boston (1997)

3. Cannarsa, P., Sinestrari, C.: Semiconcave functions, Hamilton-Jacobi equations, and optimal control, progress in nonlinear differential equations and their applications. Birkhäuser Boston Inc., Boston (2004)

4. Esteve, C., Zuazua, E.: The inverse problem for Hamilton-Jacobi equations and semiconcave envelopes. SIAM J. Math. Anal. 52, 5627-5657 (2020)

5. Evans, L.C.: Partial differential equations. The second edition, Graduate studies in mathematics, 19. American Mathematical Society, Providence (2010)

6. Ferrera, J., Gómez Gil, J.: Generalized Takagi-Van der Waerden functions and their subdifferentials. J. Convex Anal. 25, 1355-1369 (2018)

7. Fujita, Y., Hamamuki, N., Siconolfi, A., Yamaguchi, N.: A class of nowhere differentiable functions satisfying some concavity-type estimate. Acta Mathematica Hungarica 160, 343-359 (2020)

8. Fujita, Y., Hamamuki, N., Yamaguchi, N.: All the generalized characteristics for the solution to a Hamilton-Jacobi equation with the initial data of the Takagi function. SN Partial Differ. Equ. Appl. 38, 20 (2020). https://doi.org/10.1007/s42985-020-00039-7

9. Fujita, Y., Hamamuki, N., Yamaguchi, N.: A self-affine property of evolutional type appearing in a Hamilton-Jacobi flow starting from the Takagi function. Michigan Math. J. (2021). https://doi.org/10. $1307 / \mathrm{mmj} / 20195782$

10. Góra, P., Stern, R.J.: Subdifferential analysis of the Van der Waerden function. J. Convex Anal. 18, 699-705 (2011)

11. Ichihara, N., Ishii, H.: Asymptotic solutions of Hamilton-Jacobi equations with semi-periodic Hamiltonians. Comm. Partial Differ. Equ. 33, 784-807 (2008)

12. Lagarias, J.C.: The Takagi function and its properties. RIMS kôkyûroku Bessatsu B34, 153-189 (2012) 
13. Luo, S., Tran, H.V., Yu, Y.: Some inverse problems in periodic homogenization of Hamilton-Jacobi equations. Arch. Ration. Mech. Anal. 221, 1585-1617 (2016)

14. Takagi, T.: A simple example of the continuous function without derivative. Phys. Math. Soc. Japan 1, 176-177 (1903). (The Collected Papers of Teiji Takagi, S. Kuroda, Ed., Iwanami, 5-6 (1973))

Publisher's Note Springer Nature remains neutral with regard to jurisdictional claims in published maps and institutional affiliations. 